\title{
Dynamics of quasibound state formation in the driven Gaussian potential
}

\author{
Kyungsun Na, Daungruthai Jarukanont, and L. E. Reichl \\ Center for Complex Quantum Systems and Department of Physics, The University of Texas at Austin, Austin, Texas 78712, USA
}

(Received 13 March 2007; revised manuscript received 5 November 2007; published 14 April 2008)

\begin{abstract}
The quasibound states of a particle in an inverted-Gaussian potential interacting with an intense laser field are studied using complex coordinate scaling and Floquet theory. The dynamics of the driven system is different depending on whether the driving field frequency is less than or greater than the ionization frequency. As the laser field strength is increased, a new quasibound state emerges as the result of a pitchfork bifurcation in the classical phase space. Changes in the time-averaged "dressed potential" appear related to this bifurcation and provide additional confirmation of the role of the bifurcation on the emergence of a new quasibound state. The Husimi plots of the quasibound state residues reveal strong support on the periodic orbits of the bifurcation at frequencies above the ionization frequency.
\end{abstract}

DOI: 10.1103/PhysRevE.77.046208

PACS number(s): 05.45.Ac, 32.80.Fb, 05.45.Mt

\section{INTRODUCTION}

When intense laser radiation interacts with atomic and molecular systems it can produce some surprising effects. For example, laser radiation typically causes destabilization and ionization of electrons. However, if the radiation field has high enough intensity, it can actually stabilize an electron [1-8] and suppress ionization in the presence of intense laser radiation.

The key to electron stabilization is the formation of the quasibound states in the presence of radiation. Early work by Bardsley and Comella [9] showed the creation of an additional quasibound state for a particle subject to an intense laser field. Ben-Tal et al., using a time-averaged or "dressed" potential, predicted that new quasibound states could appear with increasing laser intensity in an inverted Gaussian potential [10]. Several authors have suggested that the underlying classical dynamics might provide an insight into a mechanism for the stabilization [6,7]. In this regard, the phase space distribution of the quasibound state residues have been related with the unstable periodic orbits in classical phase space [11]. The creation of a new quasibound state, with increasing laser intensity, in the presence of chaotic structures has been observed using Floquet scattering theory [12]. In this work, we show the relation between these different approaches and give evidence that one mechanism for quasibound state formation is the appearance of a bifurcation in the underlying classical phase space.

The model system studied here corresponds to a particle located in an inverted Gaussian potential and interacting with a monochromatic external field. The driven inverted Gaussian potential has been used by a number of authors $[9-13,15]$ to study the interaction of radiation with the bound states of atoms. The Hamiltonian for this system is time periodic and can be written in the form

$$
H(t)=\frac{1}{2}\left[p-\frac{\epsilon}{\omega} \sin (\omega t)\right]^{2}-V_{0} \exp \left[-(x / a)^{2}\right],
$$

where $p$ and $x$ are the momentum and position, respectively, of an electron in the presence of the inverted Gaussian potential with depth $V_{0}$ and width $a$. The external field has strength $\epsilon, \omega$ is the frequency, and $t$ is the time. All param- eters are expressed in terms of atomic units (a.u.). We will study the behavior of this system for the parameter values, $V_{0}=0.27035$ a.u. and $a=2$ a.u. These parameters describe the behavior of a one-dimensional model of the negative chlorine ion $\mathrm{Cl}^{-}$in a laser field and are also used in [12]. For these parameters only one electron bound state of energy $E_{b}=-0.1327$ a.u. is allowed in the absence of the external field. The harmonic limit of the inverted Gaussian potential has harmonic frequency $\omega_{o}=0.363$ a.u.

In our study of this driven system, we will focus on two external field frequency regimes, one below and the other above the ionization frequency $\omega_{I} \sim 0.14$. Marinescu and Gavrila [17] point out that, in these two regimes, the atom responds differently to radiation, and we also find that to be the case. We choose two external field frequencies, $\omega$ $=0.0925$ and 0.236 a.u. representing low and high frequency regimes, respectively. The high frequency case $(\omega=0.236)$ has been studied by several authors $[16,17]$ in relation with the suppression and oscillation of the ionization as the external field strength increases. In Sec. II we will investigate the classical phase space distribution for these two frequency regimes. In Sec. III we present the complex coordinate method and Floquet theory that we use to calculate the quasienergy spectrum of the driven inverted Gaussian system. In Sec. IV the classical-quantum correspondence is explored by comparing the classical and quantum phase space distributions. In Sec. V, we examine the relation between the time-averaged "dressed" potential and the distribution of the bifurcated fixed points. Conclusions are drawn in Sec. VI.

\section{CLASSICAL DYNAMICS OF THE DRIVEN GAUSSIAN SYSTEM}

The inverted Gaussian potential, when driven by a time periodic field, undergoes a transition to chaos by forming a fractal network of heteroclinic tangles in phase space in the region of influence of the inverted Gaussian. The fractal structure of the heteroclinic tangles in the classical system has been studied in great detail in $[13,14]$. Such chaotic structures have been found to support quasibound states in other systems [18-21] and some evidence has been given that unstable periodic fixed points within the chaotic struc- 


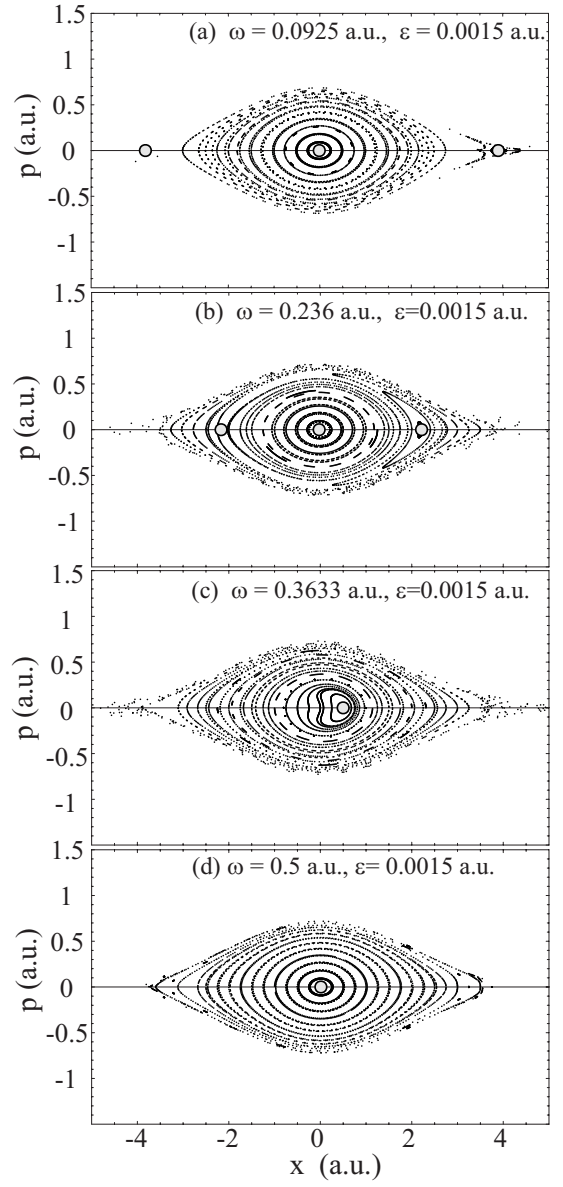

FIG. 1. Strobe plots of the system with external field strength $\epsilon=0.0015$ a.u. and (a) $\omega=0.0925$ a.u., (b) $\omega=0.236$ a.u., (c) $\omega$ $=0.3633$ a.u., and (d) $\omega=0.5$ a.u. The circles along the $p=0$ axis indicate the fixed points of the periodic orbits.

tures can support quasibound states [11]. We will give here direct evidence that periodic orbits do serve to support quasibound state structures.

For a system with one spatial dimension driven by a time periodic field, we can study the structure of orbits in the classical phase space using a Poincaré surface of section (strobe plot). Such a plot is obtained by plotting $p$ and $x$ at each period, $T=2 \pi / \omega$, of the external field [22]. It is interesting to follow the behavior of the driven system as a function of frequency. Figure 1 shows the strobe plots of classical orbits in the region of influence of the Gaussian potential with frequencies $\omega=0.0925,0.236,0.3633$, and 0.5 a.u. at field strength $\epsilon=0.0015$ a.u. The plots follow the evolution in time of a set of initial orbits. The phase space coordinates $p$ versus $x$ are plotted at times $n \frac{2 \pi}{\omega}$ where $n=0,1,2, \ldots$. At this low field strength, there is a large central island surrounded by a thin chaotic layer near the separatrix region. The open circles along the $p=0$ axis indicate the positions of key period-one periodic orbits. These orbits return to their starting points in phase space after each period of the driving field. The stable and unstable fixed points for the period-one primary resonance (which is induced by the external field) are located near $x=3.9$ and -3.81 a.u., respectively, in Fig. 1(a). The fixed point for the stable central island is near the
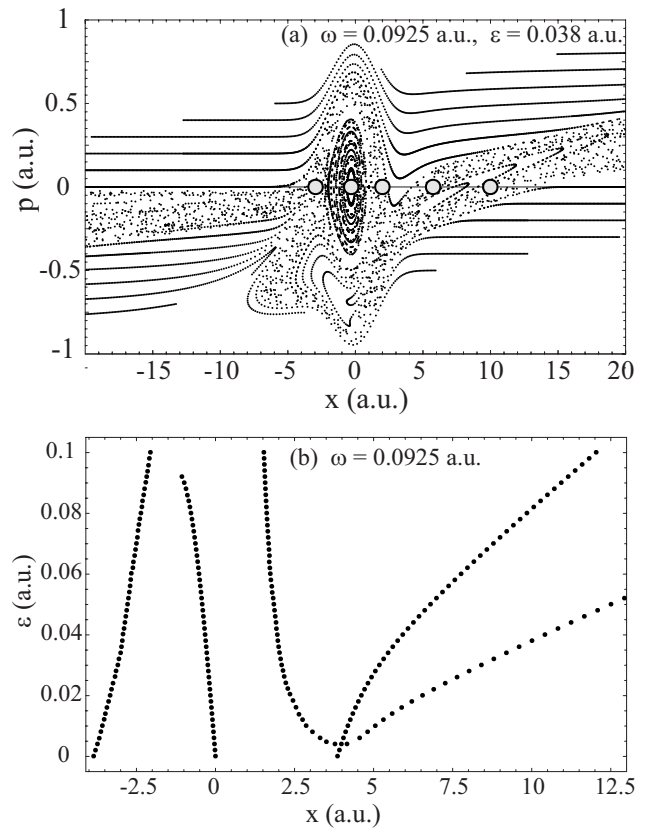

FIG. 2. (a) Strobe plot of the system with $\omega=0.0925$ a.u. and field strength $\epsilon=0.038$ a.u. The circles along the $p=0$ axis indicate the fixed points of the periodic orbits. (b) The spatial evolution of the fixed points as a function of the field strength.

origin. As we increase the external field frequency the unstable periodic orbit from the primary resonance and the periodic orbit from the central island near the origin disappear [see Fig. 1(c)]. The external field frequency $\omega=0.3633$ a.u. almost matches the natural frequency $\omega_{o}$ of the unperturbed Gaussian potential in the harmonic limit. In Fig. 1(d), the period-one periodic orbits have disappeared at the driving field frequency $\omega=0.5$ a.u. because the external field can no longer resonate with the orbits of the unperturbed Gaussian system.

In subsequent sections, we will focus on the behavior of this system for two external field frequencies, $\omega=0.0925$ and 0.236 a.u. In the remainder of this section, we consider the classical behavior of the system at these frequencies.

\section{A. Low frequency case}

Figure 2(a) shows the strobe plots with frequency $\omega$ $=0.0925$ a.u. and field strength $\epsilon=0.038$ a.u. The phase space is divided into a central island and a chaotic region surrounding that island. In addition to the interesting behavior in the region of influence of the Gaussian, we find heteroclinic tangles extending far out into the classical phase space. The size of the central island gets smaller and the heteroclinic tangles spread as the external field strength increases. The circles along the $p=0$ axis indicate the positions of key period-one periodic orbits [indicated in Fig. 2(b)] in phase space. In Fig. 2, we show the fixed points which correspond to the central island, unstable and stable fixed point pair, and the bifurcated fixed points. Figure 2(b) shows the positions of the fixed points as a function of field strength $\epsilon$ for frequency $\omega=0.0925$ a.u. At low field strength, we find several fixed points at $x<0$. The primary period-one reso- 


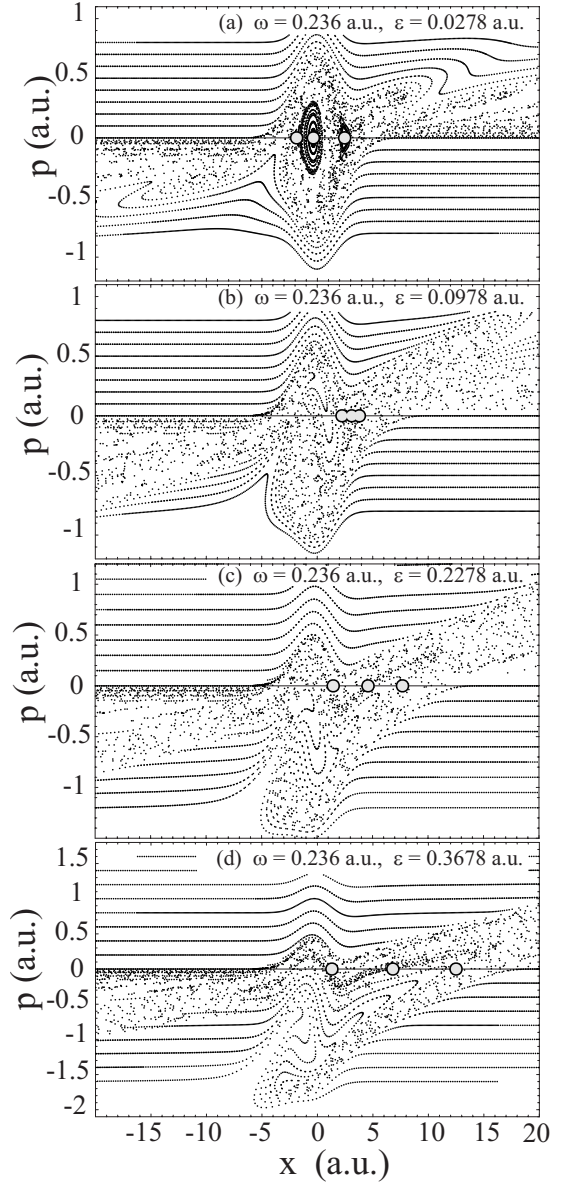

FIG. 3. Strobe plots of the system with $\omega=0.236$ a.u. at the field strengths (a) $\epsilon=0.0278$ a.u., (b) $\epsilon=0.0978$ a.u., (c) $\epsilon$ $=0.2278$ a.u., and (d) $\epsilon=0.3678$ a.u. The circles along the $p=0$ axis indicate the fixed points of the periodic orbits.

nance has a stable fixed point at $x=3.9$ a.u. and an unstable fixed point at $x=-3.81$ a.u. for $\epsilon=0.0015$ a.u. There are several other stable or unstable fixed points not shown in this figure. As we increase the field strength, all the fixed points for $x \leq 0$ a.u. (including the fixed point of the central island) disappear at about $\epsilon=0.13$ a.u. The stable fixed point of the period-one primary resonance near $x=3.9$ a.u. moves to larger values of $x$ and undergoes a pitchfork bifurcation into three period-one periodic orbits near $\epsilon=0.0041$ a.u. Note that in Fig. 2(a), the fixed points are separated by regions in the tangle structure that originate in the asymptotic region of the phase space.

For this low frequency, the bifurcation occurs at a field strength lower than the field strength where the other fixed points disappear. We will find qualitatively different behavior for the high frequency case.

\section{B. High frequency case}

Figure 3 shows strobe plots for the system driven with frequency $\omega=0.236$ a.u. and field strengths $\epsilon=0.0278$, $0.0978,0.2278$, and 0.3678 a.u. At the field strength $\epsilon$ $=0.0278$ a.u. in Fig. 4(a), the period-one primary resonance has a stable fixed point at $x=2.43$ a.u. and an unstable fixed

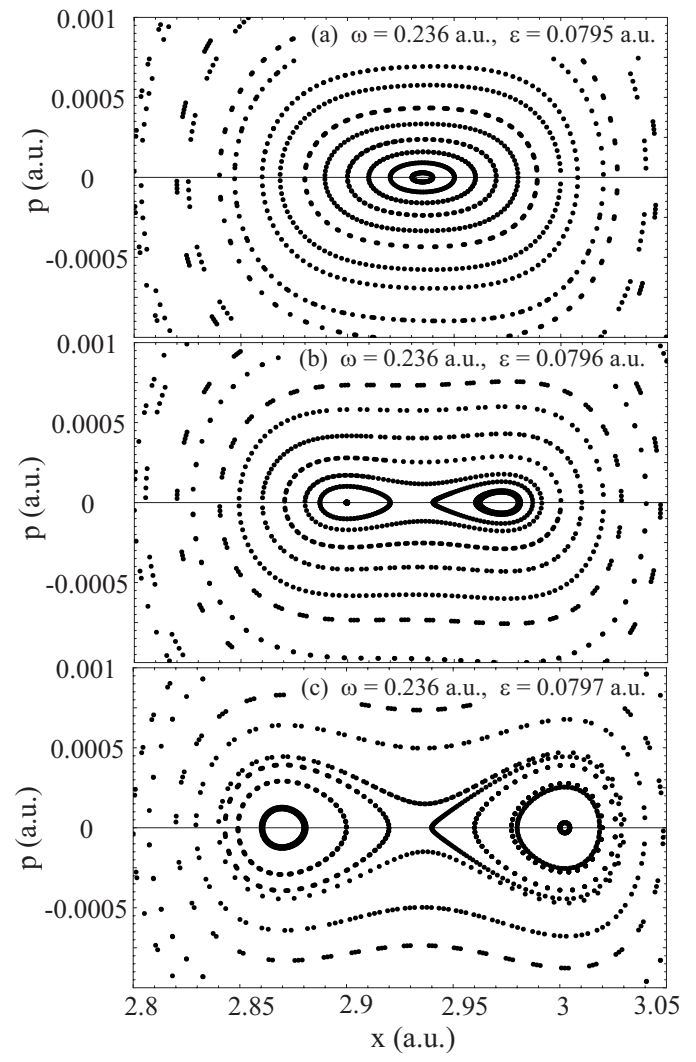

FIG. 4. Strobe plots of the system with $\omega=0.236$ a.u. and (a) $\epsilon=0.0795$ a.u., (b) $\epsilon=0.0796$ a.u., and (c) $\epsilon=0.0797$ a.u. The circles along the $p=0$ axis indicate the fixed points of the periodic orbits.

point at $x=-2.0$ a.u. The fixed point of the central island is at $x=-0.32$ a.u. The unstable fixed point of the primary resonance and the fixed point of the central island have disappeared for the field intensity near $\epsilon=0.0978$ in Fig. 3(b), and the stable fixed point of the primary resonance has undergone a bifurcation. Note that the fixed points of the bifurcation remain connected by chaotic tangles. Also note that the heteroclinic tangles and the bifurcated fixed points move apart in Figs. 3(c) and 3(d) with increasing field intensity.

In Fig. 4 we focus on the pitchfork bifurcation which occurs at about $\epsilon=0.0796$. The period-one stable fixed points are created and move apart. As the field strength increases further, these two newly created period-one stable fixed points themselves become unstable and undergo a sequence of period-doubling bifurcations. These additional bifurcations occur on a very small scale in the phase space.

Figure 5 shows the positions of the bifurcated period-one fixed points as a function of field strength $\epsilon$ for the frequency $\omega=0.236$ a.u. As the external field intensity increases the unstable fixed point of the period-one primary resonance and the fixed point of the central island approach and disappear near the intensity $\epsilon=0.06$ a.u. The stable fixed point of the period-one primary resonance moves to larger values of $x$ and bifurcates into three fixed points near $\epsilon=0.0796$ a.u. As we will see later, the spatial distribution of the bifurcated fixed points is closely related with the quantum probability distribution of the stabilized quasibound states of the system. 


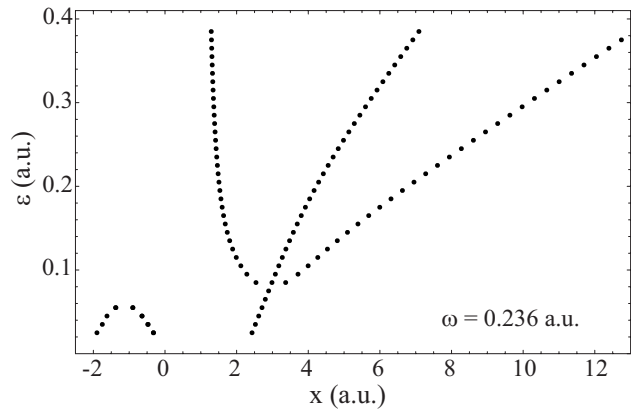

FIG. 5. The spatial evolution of the fixed points as a function of the field strength for the system with $\omega=0.236$ a.u.

\section{QUANTUM DYNAMICS OF THE DRIVEN GAUSSIAN SYSTEM}

When the external field is applied to the inverted Gaussian system, bound states become quasibound states with finite lifetime. We use smooth exterior scaling (SES) [23,24] in order to determine the lifetime of quasibound states. This approach rotates the coordinate $x$ but only in the region outside the influence of the potential. It allows us to obtain an undistorted view of the phase space distribution of the residues associated with the quasibound states.

The basic idea of SES is to scale the $x$ coordinate by the factor $e^{i \theta}$, but only in the region $|x| \geq x_{s}$ where the potential is zero. A discontinuity in the scaling at $x= \pm x_{s}$ is avoided by using a smoothed scaling relation $x \rightarrow \tilde{x}$ where

$$
x \rightarrow \tilde{x}=x+\left(e^{i \theta}-1\right)\left[x+\frac{1}{2 \lambda} \ln \left(\frac{\cosh \left[\lambda\left(x-x_{s}\right)\right]}{\cosh \left[\lambda\left(x+x_{s}\right)\right]}\right)\right] .
$$

In this paper, we use $\lambda=5$ and $x_{s}=25$ a.u. unless otherwise stated. The scaled coordinate $\tilde{x} \rightarrow x$ for $|x| \leq x_{s}$ and $\tilde{x} \rightarrow x e^{i \theta}$ for $x \rightarrow \infty$.

Under this transformation, the Hamiltonian for the scaled undriven inverted Gaussian system takes the form

$$
\tilde{H}_{0}(x)=\frac{p^{2}}{2}-V_{0} \exp \left[-(x / a)^{2}\right]+V(x)
$$

where

$$
V(x)=\frac{\ddot{f}}{4 f^{3}}-\frac{5 \dot{f}^{2}}{8 f^{4}}+\frac{\dot{f}}{f^{3}} \frac{\partial}{\partial x}+\frac{1}{2}\left(1-\frac{1}{f^{2}}\right) \frac{\partial^{2}}{\partial x^{2}},
$$

and $f(x)=\frac{\partial \widetilde{x}}{\partial x}$ and $\dot{f}=\frac{d f}{d x}$.

We use the particle-in-a-box states for the basis states,

$$
\langle x \mid n\rangle=\sqrt{\frac{2}{L}} \sin \left(\frac{n \pi x}{L}-\frac{n \pi}{2}\right),
$$

where $-L / 2 \leq x \leq L / 2$. The eigenstates of the scaled Hamiltonian $\widetilde{H}_{0}$ can be expanded in terms of these basis states so that

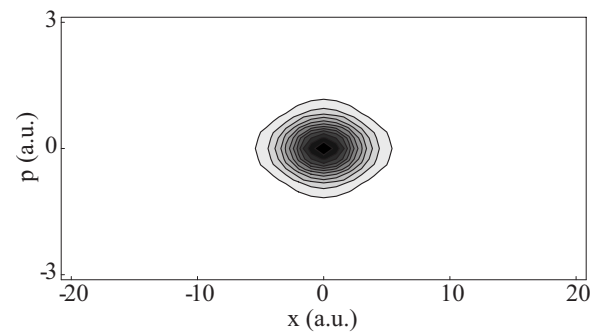

FIG. 6. Husimi distribution of the bound state of the unperturbed inverted Gaussian system using $\sigma=2$ a.u.

$$
\left|\psi_{i}\right\rangle=\sum_{n=1}^{N} c_{n i}|n\rangle
$$

Note that because the scaled Hamiltonian is not Hermitian, the states $\left|\psi_{i}\right\rangle=\sum_{n=1}^{N} c_{i n}|n\rangle$ and $\left\langle\psi_{i}\right|=\sum_{n=1}^{N} c_{i n}\langle n|$ satisfy a bilinear normalization condition $\sum_{n=1}^{N} c_{i n}^{2}=1$. In this study we have achieved a numerical convergence with the number of basis states $N=200$ and the size of the system $L=200$ a.u.

We use Husimi plots to visualize the distribution of probability, in phase space, of quasibound state residues in the region of influence of the Gaussian potential. The Husimi function $G(x, p)$ for any state $\psi(x)$ can be written [25]

$$
G(x, p)=\frac{1}{2 \pi}\left|\left(\frac{1}{2 \pi \sigma^{2}}\right)^{1 / 4} \int_{-\infty}^{\infty} e^{\left(x^{\prime}-x\right)^{2} / 2 \sigma^{2}-i p x^{\prime}} \psi\left(x^{\prime}\right) d x^{\prime}\right|^{2},
$$

where $\sigma$ is a coarse-graining parameter that determines the width of the Gaussian in the $x$ and $p$ directions. A Husimi plot of the one bound state of our inverted Gaussian system is shown in Fig. 6 for $\sigma=2$ a.u. The dispersions in spatial and momentum distribution of the wave packet are $\sigma_{x}$ $=\sqrt{\sigma / 2}=1.41$ a.u. and $\sigma_{p}=\sqrt{1 / 2 \sigma}=0.35$ a.u., respectively.

We study the quasibound states in the quantum system using Floquet theory together with SES. Because the scaled driven Hamiltonian $\tilde{H}(t)$ is time-periodic, the solutions, $|\psi(t)\rangle$, to the Schrödinger equation

$$
i \frac{\partial|\psi(t)\rangle}{\partial t}=\tilde{H}(t)|\psi(t)\rangle
$$

can be expanded in Floquet eigenstates [which we denote as $\left.\left|\phi_{\alpha}(t)\right\rangle\right]$ so that

$$
|\psi(t)\rangle=\sum_{\alpha} A_{\alpha} e^{-i \Omega_{\alpha} t}\left|\phi_{\alpha}(t)\right\rangle
$$

The Floquet eigenstates satisfy the eigenvalue equation

$$
\left[\tilde{H}(t)-i \frac{\partial}{\partial t}\right]\left|\phi_{\alpha}(t)\right\rangle=\Omega_{\alpha}\left|\phi_{\alpha}(t)\right\rangle
$$

where $\Omega_{\alpha}$ is the $\alpha$ th Floquet eigenphase. The Floquet eigenstates $\left|\phi_{\alpha}(t)\right\rangle$ are time-periodic $\left|\phi_{\alpha}(t)\right\rangle=\left|\phi_{\alpha}(t+T)\right\rangle$, where $T$ is the period of the Hamiltonian, and they form a complete orthonormal set. At the time $t=T$, the solution to the Schrödinger equation can be written 


$$
|\psi(T)\rangle=\sum_{\alpha} e^{-i \Omega_{\alpha} T}\left|\phi_{\alpha}(0)\right\rangle\left\langle\phi_{\alpha}(0) \mid \psi(0)\right\rangle .
$$

From Eq. (11), the Floquet evolution operator at time $t=T$ is defined as

$$
\hat{U}(T)=\sum_{\alpha} e^{-i \Omega_{\alpha} T}\left|\phi_{\alpha}(0)\right\rangle\left\langle\phi_{\alpha}(0)\right| .
$$

The eigenvalues and eigenstates of the Floquet evolution operator $\hat{U}(T)$ can be computed numerically by evaluating $\hat{U}(T)$ using the eigenstates, $\left|\psi_{i}\right\rangle$, of $\widetilde{H}_{0}$, so that

$$
U_{i, j}(T)=\sum_{\alpha} e^{-i \Omega_{\alpha} T}\left\langle\psi_{i} \mid \phi_{\alpha}(0)\right\rangle\left\langle\phi_{\alpha}(0) \mid \psi_{j}\right\rangle .
$$

If we diagonalize the Floquet matrix in Eq. (13), we obtain the eigenvalues $e^{-i \Omega_{\alpha} T}$ and coefficients $c_{i}$ in the eigenstate expansion

$$
\left|\phi_{\alpha}(T)\right\rangle=\left|\phi_{\alpha}(0)\right\rangle=\sum_{i} c_{i}\left|\psi_{i}\right\rangle .
$$

The Floquet matrix in Eq. (13) is computed by writing the Schrödinger equation in terms of a basis set composed of the eigenstates $\left|\psi_{i}\right\rangle$. We then integrate the Schrödinger equation for one period of time using one of the eigenstates $\left|\psi_{i}\right\rangle$ as an initial condition. This gives one column of the Floquet time evolution matrix. This process is repeated for each eigenstate until the full Floquet matrix is constructed.

The time-dependent scaled Schrödinger equation for the driven inverted Gaussian system is

$$
\begin{aligned}
i \frac{\partial}{\partial t}|\Psi(t)\rangle= & \widetilde{H}_{0}|\Psi(t)\rangle-\frac{\epsilon}{\omega} \tilde{p} \sin (\omega t)|\Psi(t)\rangle \\
& +\frac{\epsilon^{2}}{2 \omega^{2}} \sin ^{2}(\omega t)|\Psi(t)\rangle,
\end{aligned}
$$

where $\tilde{p}$ is the complex scaled momentum operator. We can calculate the matrix element of $\tilde{p}$ in terms of the unperturbed eigenstates of $\widetilde{H}_{0}$, so

$$
\left\langle\psi_{i}|\tilde{p}| \psi_{j}\right\rangle=\sum_{m=1}^{N} \sum_{n=1}^{N} c_{m i} c_{n j}\langle m|\tilde{p}| n\rangle,
$$

where we use the fact that $\left|\psi_{i}\right\rangle$ is expanded in terms of $|n\rangle$.

As described in [23], the eigenvalues $e^{-i \Omega_{\alpha} T}$ will consist of continuum states and quasibound states, all of which lie inside or on the unit circle. The continuum states change position as the scaling angle $\theta$ is changed. The quasibound states are independent of $\theta$ and do not change position as $\theta$ is changed. By changing $\theta$ and observing the positions of the various eigenvalues $e^{-i \Omega_{\alpha} T}$, we can determine which eigenvalues correspond to quasibound states.

\section{QUASIBOUND STATES}

We now examine the quasibound state structure of the driven inverted Gaussian system. We first consider the behavior of quasibound states for the low frequency $\omega$ $=0.0925$ a.u. for varying field strengths. Then we consider

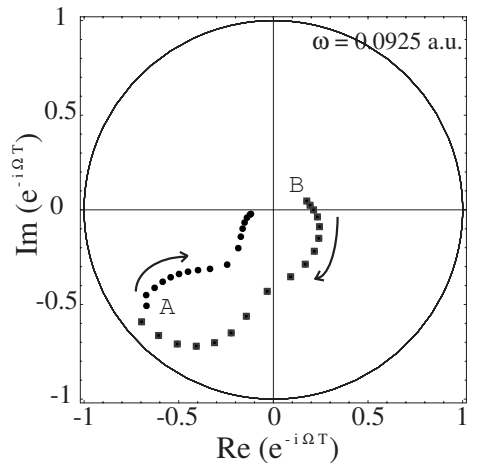

FIG. 7. Quasibound states for the field frequency $\omega$ $=0.0925$ a.u. with varying external field strengths from $\epsilon=0.034$ to 0.050 a.u.

the quasibound state behavior for the high frequency, $\omega$ $=0.236$, and compare the behavior of quasibound states in these two dynamical regimes. We will find that they behave very differently.

\section{A. Low frequency case}

Figure 7 shows the eigenvalues $e^{-i \Omega_{\alpha} T}$ associated with quasibound states (we do not show continuum states in this plot) for the field frequency $\omega=0.0925$ a.u. with external field strengths varying from $\epsilon=0.034$ to 0.05 a.u. These eigenvalues were obtained by using SES with $\theta=0.3$ and $x_{s}$ $=25$ a.u. The quasibound state (called $A$ ) that emerges from the bound state of the inverted Gaussian is located at point $A$ at $\epsilon=0.034$ a.u. and moves in the direction of the arrow as the external field strength increases to $\epsilon=0.50$ a.u. As it moves, it becomes increasingly unstable. A second quasibound state (called $B$ ) originates from the continuum with very short lifetime and is located at point $B$ for $\epsilon=0.034$. As $\epsilon$ increases from $\epsilon=0.034$ to 0.50 a.u. it follows the arrow and becomes increasingly more stable.

The lifetime of the quasibound states can be evaluated by writing the eigenphase $\Omega_{\alpha}$ in the form $\Omega_{\alpha}=q_{\alpha}-i \Gamma_{\alpha} / 2$, where $\tau_{\alpha}=1 / \Gamma_{\alpha}$ is the lifetime of the quasibound state. The lifetime for each state is given in multiples of the driving field period $T=2 \pi / \omega$. At $\epsilon=0.034$, the quasibound state $A$ has a lifetime $\tau=2.88 \mathrm{~T}$ and the quasibound state $B$ has a lifetime $\tau$ $=0.29 \mathrm{~T}$. The two quasibound states undergo an avoided crossing at $\epsilon=0.043$ a.u. After the avoided crossing between the two quasibound states occurs, the quasibound state $B$ approaches the unit circle and becomes long-lived with lifetime, $\tau=5.5 \mathrm{~T}$, while the quasibound state $A$ moves away from the unit circle and becomes short-lived with lifetime, $\tau=0.23 \mathrm{~T}$.

The phase space distributions of quasibound state residues associated with these eigenvalues exchange their character as they undergo the avoided crossing. Figure 8 shows the Husimi distributions for the residues of quasibound states $A$ and $B$ for field strengths $\epsilon=0.038,0.043,0.045$, and 0.050 a.u. The change in quasibound state $A$ as the field strength increases is shown in Figs. 8(a1)-8(a4). The Husimi distribution of this quasibound state at $\epsilon=0.038$ a.u. has a structure similar to the bound state of the unperturbed system shown 


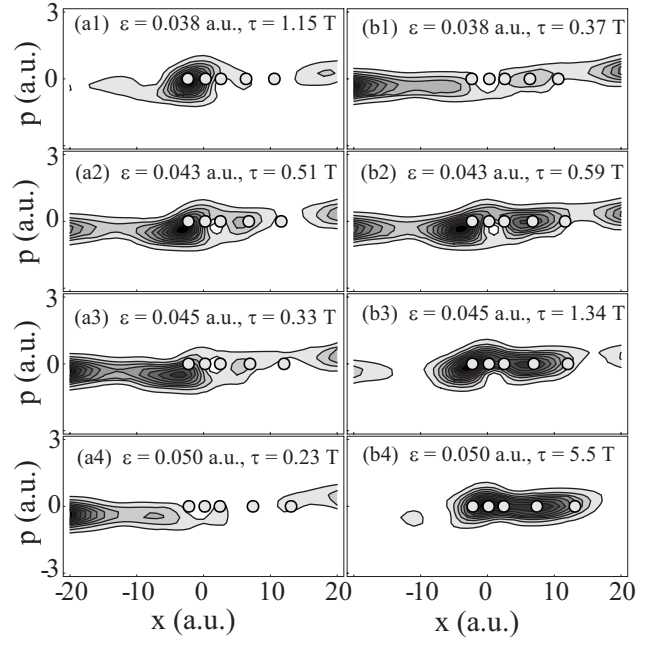

FIG. 8. Husimi distributions of the quasibound states at the frequency $\omega=0.0925$ a.u. with field strengths $\epsilon=0.038,0.043,0.045$, and 0.050 a.u. from top to bottom. (a1)-(a4) show a variation of the quasibound state originated from the bound-state as the field strength increases. (b1)-(b4) show a variation of the quasibound state originated from the continuum as the field strength increases.

in Fig. 6. As the field strength increases, the Husimi distribution develops an elongated structure toward the continuum. The change in quasibound state $B$ is shown in Figs. 8(b1)-8(b4). The Husimi distribution of this quasibound state at $\epsilon=0.038$ a.u. is strongly coupled to the continuum. The Husimi distributions for these two quasibound states near the avoided crossing at $\epsilon=0.043$ a.u. contain a superposition of both the bound statelike structure and strong continuum component. After the avoided crossing occurs, the Husimi distribution of quasibound state $A$ keeps spreading out into the continuum and the Husimi distribution of quasibound state $B$ develops contracted structure near the origin as the field strength increases. The circles along the $p=0$ axis show the fixed points of key periodic orbits in classical phase space. The probability distribution of the quasibound states with long lifetimes are concentrated on the fixed points of the bifurcation in the underlying classical phase space.

Quasibound state $B$ emerges from the continuum at about $\epsilon=0.038$ which is a field strength well above the classical bifurcation. At $\epsilon=0.038$, the classical phase space contains a large chaotic region formed from heteroclinic tangles in addition to the fixed points from the bifurcation. The emerging quasibound state is exposed to a large underlying chaotic sea classically and has strong coupling to the continuum giving it a fairly short lifetime. A similar phenomenon has been reported in an electron waveguide system [18]. In the next section, we find that the quasibound states, for the high frequency case, have very different behavior.

\section{B. High frequency case}

Figure 9 shows the continuum (closed circles) and quasibound state (gray circles) eigenvalues $e^{i \Omega_{\alpha} T}$ for external field strengths $\epsilon=0.0278,0.0978,0.2278$, and 0.3678 a.u. These were obtained by using SES with $\theta=0.3$ and $x_{s}=20$ a.u. At

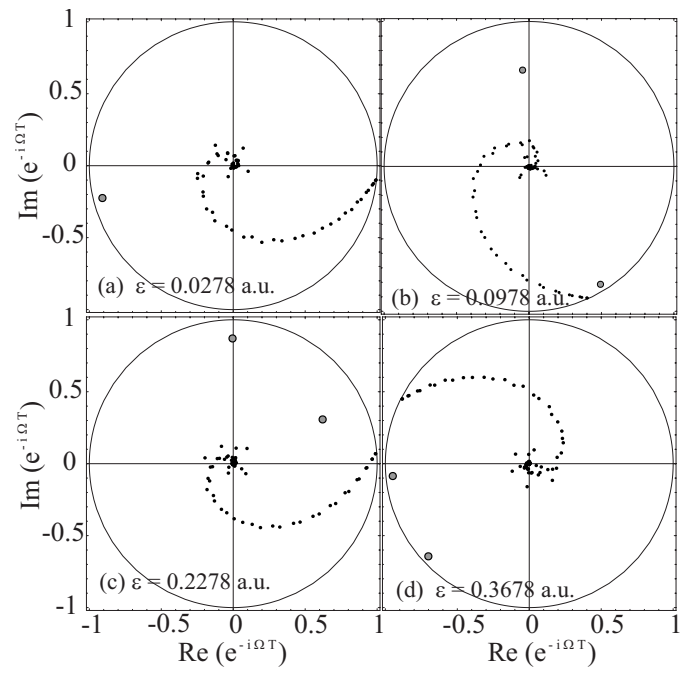

FIG. 9. Eigenvalues for the field frequency $\omega=0.236$ a.u. with external field strengths at (a) $\epsilon=0.0278$ a.u., (b) $\epsilon=0.0978$ a.u., (c) $\epsilon=0.2278$ a.u., and (d) $\epsilon=0.3678$ a.u. Continuum (closed circles) and quasibound state poles (gray circles) are shown.

low field intensity, $\epsilon=0.0278$ a.u., in Fig. 9(a) there is one quasibound state $A$ which is the destabilized ground state of the inverted Gaussian potential.

As field strength increases, a new quasibound state emerges from the continuum states near the unit circle at about $\epsilon \approx 0.0978$ [see Fig. 9(b)]. The state shown in Fig. 9(b) is a quasibound state because it has the property that its position in the unit circle does not change as the scaling angle $\theta$ is changed, whereas the positions of continuum states do change. Note that this criterion for identifying quasibound states becomes less accurate for states near the unit circle because the dependence of the continuum states on the scaling angle $\theta$ becomes weaker close to the unit circle. With the numerical accuracy available to us, we can determine the value of $\epsilon$ at which this state does emerge to an accuracy of plus or minus 0.01 a.u.

Once the new quasibound state $B$ has emerged near the unit circle, the two quasibound states move around inside the unit circle as we increase the external field strength. However, unlike the low frequency case, these two quasibound states do not undergo an avoided crossing. Both of them approach the unit circle and attain long lifetimes, $\tau=10.22$ and $10.15 \mathrm{~T}$ at $\epsilon=0.3678$ a.u. in Fig. 9(d). Thus the lifetimes of both quasibound states increases with increasing external field strength, indicating that stabilization of the electron has occurred. This field strength seems to agree with the field strength where the first minimum of the ionization occurs in [16] and the Born approximation of the high-frequency Floquet theory of [17].

The Husimi distributions of the quasibound state residues at frequencies corresponding to those in Fig. 9 are shown in Fig. 10. The Husimi distribution for quasibound state $A$ at $\epsilon=0.0278$ a.u. has a structure similar to the bound state of the inverted Gaussian system. The evolution in the phase space distribution of quasibound state $A$ is shown in Figs. 10(a1)-10(a4). Figures 10(b1)-10(b3) show the probability distribution of quasibound state $B$ with increasing $\epsilon$. As field 


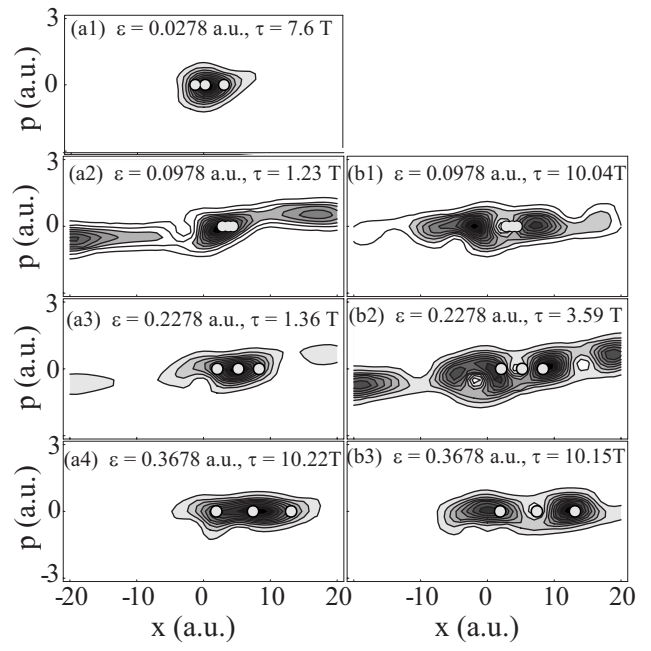

FIG. 10. Husimi distributions of the quasibound states at the frequency $\omega=0.236$ a.u. with field strengths $\epsilon=0.0278,0.0978$, 0.2278 , and 0.3678 a.u. from top to bottom. (a1)-(a4) show a variation of the quasibound state originated from the bound-state as the field strength increases. (b1)-(b4) show a variation of the quasibound state originated from the continuum as the field strength increases.

strength increases, both quasibound states develop contracted structure on the fixed points of the bifurcation.

It is interesting to compare the behavior of this system with that of a particle in Morse potential (not shown here) $[10,19]$, with the same eigenvalue distribution, and driven by a time-periodic external field. For the Morse system, the stable fixed point of the period-one primary resonance does not undergo a bifurcation and, for all values of $\epsilon$, there exists only one quasibound state corresponding to the bound state for the unperturbed system. In the next section, we give further evidence that the appearance of quasibound state $B$ is associated with the bifurcation.

\section{DRESSED INVERTED GAUSSIAN POTENTIAL}

The dressed inverted Gaussian potential is obtained by first transforming to the Kramers-Henneberger (KH) frame of reference [12] (a coordinate frame that moves with the electron). In the $\mathrm{KH}$ frame, the inverted Gaussian potential oscillates back and forth with the frequency of the driving field. A dressed potential $\bar{V}(x)$ is then obtained by averaging oscillating inverted Gaussian potential over one period of the oscillation such that

$$
\bar{V}(x)=-V_{0} \int_{0}^{1} \exp \left\{-\left[\left(x+\frac{\epsilon}{\omega^{2}} \cos \left(2 \pi t^{\prime}\right)\right) / a\right]^{2}\right\} d t^{\prime} .
$$

At low external field strength $\epsilon$, the dressed potential is similar to the inverted Gaussian with only one minimum. However, as the external field strength increases, the dressed potential changes shape and takes the form of a double well potential. This was used in Ref. [10] as the reason that a new quasibound state appears in the driven inverse Gaussian po-

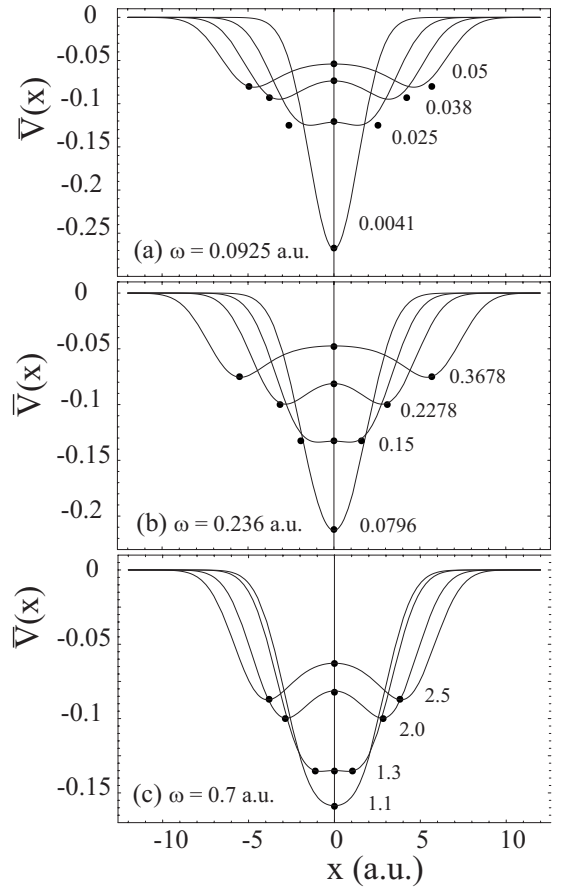

FIG. 11. Average inverted Gaussian potential superimposed with the positions of the bifurcated fixed points for (a) the low frequency case with $\omega=0.0925$ a.u. and (b) the high frequency case with $\omega=0.0236$ a.u., and (c) $\omega=0.7$ a.u. External field strengths $(\epsilon)$ are indicated inside. The positions of the fixed points are shifted in accordance with the position of the central unstable fixed point to be at the origin.

tential. Such a change in shape does not occur in the driven Morse system and no new quasibound states appear in the Morse system. It is worth noting that the dressed potential approach for a system driven by a periodic perturbation has been used to show the appearance of a new stable state for the inverted pendulum [26].

Figure 11(a) shows the dressed potential for the inverted Gaussian system for external field frequency $\omega$ $=0.0925$ a.u. and field strengths $\epsilon=0.0041,0.0025,0.038$, and 0.050 a.u. As the external field strength increases, the depth of the dressed potential decreases, as was pointed out in [10]. Black dots on the potential give the position of fixed points associated with the bifurcation, with the central fixed point always placed at the central extremum of the dressed potential. Note that the positions of the stable fixed points lie at or near the minima of the dressed potential. In classical phase space, the bifurcation starts near the field strength $\epsilon$ $=0.0041$ a.u. The double well structure in the dressed potential begins to form near $\epsilon=0.025$ a.u. The short-lived quasibound state emerges near $\epsilon=0.038$ a.u. Thus at low frequencies the dressed potential does not accurately track the underlying dynamics, as is well-known.

Figure 11(b) shows the dressed potential for frequency $\omega=0.236$ a.u. and field strengths $\epsilon=0.0796,0.15,0.2278$, and 0.3678 a.u. Again, the depth of the dressed potential decreases as the external field strength increases. For this high frequency case, the two stable fixed points of the bifurcation lie closer to the minima of the double well than was the case at low frequency. The bifurcation occurs at $\epsilon$ 


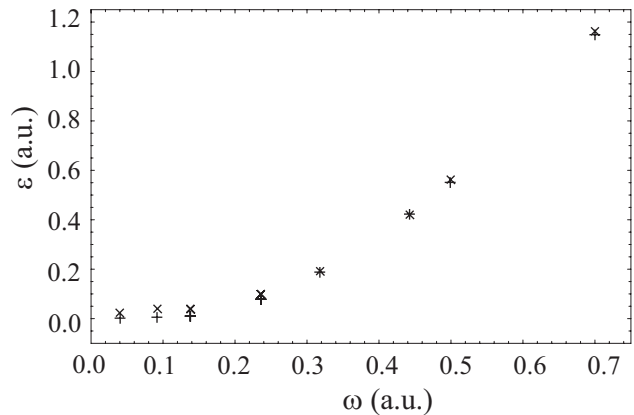

FIG. 12. The minimum external field strength where the bifurcation in classical phase space occurs and the quasibound state begins to form, as a function of the frequency. The emergence of the classical bifurcation and the formation of the quasibound state are marked by "+" and " $\times$," respectively.

$=0.0796$ and the new quasibound state emerges at about $\epsilon$ $=0.0978$.

As we increase the external field frequency, the positions of the stable fixed points of the bifurcation and the positions of the minima of the dressed inverted Gaussian potential begin to coincide almost exactly. In Fig. 11(c), we show the dressed potential and the fixed points of the bifurcation for external field frequency $\omega=0.7$ a.u.

We have done this same analysis for the deeper inverted Gaussian potential used in [10]. In that case, $V_{0}=0.63, a$ $=2.65$ a.u., external field frequency is $\omega=0.0925$ a.u., and a variety of field strengths. We again find this same good correspondence between the positions of the fixed points of the bifurcation and the minima of the dressed potential. All these results indicate that the change in shape of the dressed potential from a single well to a double well potential, as external field strength increases, is a result of the bifurcation in the underlying dynamics. The higher the frequency of the driving field and the deeper the potential, the better the correspondence.

In Fig. 12 we show the field strength at which the bifurcation occurs as a function of frequency (marked by "+"). In the same figure we show the field strength at which the qua- sibound state emerges (marked by " $X$ "). The classical bifurcation, as a function of field intensity, always occurs before the quasibound state forms. The agreement is very good. We also find that the underlying classical phase space evolves rapidly from regular to chaotic structure as the external field strength is increased at low frequency. The same development of the phase space structure can be observed over a much wider range of external field strength at high frequency.

\section{CONCLUSIONS}

The Floquet spectrum of the driven inverted Gaussian system has been calculated using complex coordinate rotation. The emergence and movement of quasibound states in the unit circle have been studied in order to understand the mechanism for stabilization of the system with an increasing external field intensity. The quantum-classical correspondence is investigated by comparing Husimi distributions of quasibound state residues to classical phase space structures. The number of quasibound states increases from one to two as field intensity increases. We find distinctly different dynamics depending on whether the driving frequency is below or above the ionization frequency.

For the low frequency case, the complex eigenvalues $e^{-i \Omega_{\alpha} T}$ associated with the quasibound states undergo an avoided crossing as we vary the field strength. For this case, the new quasibound state emerges with very short lifetime and its residue, as it emerges, has partial support on the classical chaotic tangles and is strongly connected to the continuum.

For the high frequency case, the residue of the new emerging quasibound state has support on the periodic orbits of the bifurcation and these periodic orbits are fully immersed in the chaotic tangles associated with the reaction region. As we increase the laser intensity for the high frequency case, the residues of the two quasibound states become contracted into regions near the fixed points of the bifurcation, thereby stabilizing the electron-chlorine ion system.
[1] M. P. deBoer, J. H. Hoogenraad, R. B. Vrijen, L. D. Noordam, and H. G. Muller, Phys. Rev. Lett. 71, 3263 (1993).

[2] M. P. deBoer, J. H. Hoogenraad, R. B. Vrijen, R. C. Constantinescu, L. D. Noordam, and H. G. Muller, Phys. Rev. A 50, 4085 (1994).

[3] Q. Su, J. H. Eberly, and J. Javanainen, Phys. Rev. Lett. 64, 862 (1990).

[4] Q. Su and J. H. Eberly, J. Opt. Soc. Am. B 7, 564 (1990).

[5] J. H. Eberly and K. C. Kulander, Science 262, 1229 (1993).

[6] R. Grobe and C. K. Law, Phys. Rev. A 44, R4114 (1991).

[7] R. V. Jensen, M. M. Sanders, M. Saraceno, and B. Sundaram, Phys. Rev. Lett. 63, 2771 (1989); B. Sundaram and R. V. Jensen, Phys. Rev. A 47, 1415 (1993).

[8] A. T. Rosenberger, C. C. Sung, S. D. Pethel, and C. M. Bowden, Phys. Rev. A 56, 2459 (1997).
[9] J. N. Bardsley and M. J. Comella, Phys. Rev. A 39, 2252 (1989).

[10] N. Ben-Tal and N. Moiseyev, J. Chem. Phys. 98, 9610 (1993).

[11] T. Timberlake and L. E. Reichl, Phys. Rev. A 64, 033404 (2001).

[12] A. Emmanouilidou and L. E. Reichl, Phys. Rev. A 65, 033405 (2002).

[13] A. Emmanouilidou, C. Jung, and L. E. Reichl, Phys. Rev. E 68, 046207 (2003).

[14] A. Emmanouilidou and C. Jung, Phys. Rev. E 73, 016219 (2006).

[15] N. Ben-Tal, N. Moiseyev, R. Kosloff, and C. Cerjan, J. Phys. B 26, 1445 (1993); T. Timberlake and L. E. Reichel, Phys. Rev. A 64, 033404 (2001).

[16] G. Yao and Shih-I. Chu, Phys. Rev. A 45, 6735 (1992). 
[17] M. Marinescu and M. Gavrila, Phys. Rev. A 53, 2513 (1996).

[18] H. Lee, C. Jung, and L. E. Reichl, Phys. Rev. B 73, 195315 (2006).

[19] D. Jarukanont, K. Na, and L. E. Reichl, Phys. Rev. A 75, 023403 (2007).

[20] T. Timberlake, F. Petruzielo, and L. E. Reichl, Phys. Rev. E 72, 016208 (2005).

[21] E. Wigner, Phys. Rev. 40, 749 (1932).

[22] L. E. Reichl, The Transition to Chaos: Conservative Classical Systems and Quantum Manifestations, 2nd ed. (Springer-
Verlag, Berlin, 2004).

[23] W. P. Reinhardt, Annu. Rev. Phys. Chem. 33, 223 (1982).

[24] N. Moiseyev, Phys. Rep. 302, 212 (1998); J. Phys. B 31, 1431 (1998).

[25] K. Takahashi, Prog. Theor. Phys. 98 (Suppl.), 109 (1989); Y. Chun and H. Lee, Ann. Phys. 307, 438 (2003).

[26] L. D. Landau and E. M. Lifshitz, Mechanics, 3rd ed. (Pergamon, London, 1981); R.Z. Sagdeev, D.A. Usikov, and G.M. Zaslavsky, Nonlinear Physics (Harwood, London, 1988). 